\title{
BMJ Open Perioperative management for people with chronic kidney disease receiving dialysis undergoing major surgery: a protocol for a scoping review
}

\author{
Tyrone G Harrison (10 , ,2 Brenda R Hemmelgarn,, ${ }^{1,3}$ Janine F Farragher, ${ }^{2}$ \\ Connor O'Rielly, ${ }^{1,2}$ Maoliosa Donald, ${ }^{2}$ Matthew James, ${ }^{1,2,4,5}$ Deirdre McCaughey, ${ }^{2,4}$ \\ Shannon M Ruzycki (D , , ${ }^{1,2}$ Kelly B Zarnke, ${ }^{1,4}$ Paul E Ronksley ${ }^{2,4}$
}

To cite: Harrison TG, Hemmelgarn BR, Farragher JF, et al. Perioperative management for people with chronic kidney disease receiving dialysis undergoing major surgery: a protocol for a scoping review. BMJ Open 2020;10:e038725. doi:10.1136/ bmjopen-2020-038725

- Prepublication history for this paper is available online. To view these files, please visit the journal online (http://dx.doi. org/10.1136/bmjopen-2020038725).

Received 21 March 2020 Revised 15 August 2020 Accepted 18 August 2020

Check for updates

(C) Author(s) (or their employer(s)) 2020. Re-use permitted under CC BY-NC. No commercial re-use. See rights and permissions. Published by BMJ.

For numbered affiliations see end of article.

Correspondence to Dr Paul E Ronksley; peronks|@ucalgary.ca

\section{ABSTRACT}

Introduction People with chronic kidney disease receiving dialysis (CKD G5D) have an increased risk of poor postoperative outcomes and a high incidence of major surgery. Despite the high burden of these combined risks, there is a paucity of evidence to support tailored perioperative strategies to manage this population. A comprehensive evidence synthesis would inform the management of these patients in the perioperative period and identify knowledge gaps. We describe a protocol for a scoping review of the literature to identify existing perioperative strategies, protocols, pathways and interventions for people with CKD G5D undergoing major surgery.

Methods and analysis We will conduct a scoping review in accordance with the Joanna Briggs Institute methodology and report per the Preferred Reporting Items for Systematic Reviews and Meta-Analyses extension for scoping reviews. In February 2020, we will complete our search of MEDLINE, EMBASE, CINAHL Plus, Cochrane Database of Systematic Reviews, and Cochrane Controlled Trials Registry for published literature from inception to present. All study types are eligible for inclusion, without language restriction. Studies reporting a perioperative intervention in adult patients with CKD G5D are eligible for inclusion. Studies in prevalent kidney transplant patients or patients with acute kidney injury, and studies that report on surgical approaches without consideration of perioperative management strategies, will be excluded. Reviewers will independently assess abstracts for all identified studies in duplicate, and again at the full-text stage. Following published literature searches, a search of the grey literature will be developed. We will extract and narratively report study, participant and intervention details. This will include a summary table outlining the strategies employed, organised into post hoc developed perioperative domains.

Ethics and dissemination Ethical considerations do not apply to this scoping review. Findings will be disseminated through relevant conference presentations and publications.

\section{INTRODUCTION}

Chronic kidney disease (CKD) is generally defined based on laboratory measures of estimated glomerular filtration rates (eGFR) or abnormalities in kidney structure or urine content, ${ }^{1}$ and affects $10 \%-15 \%$ of adults in developed countries. ${ }^{2}$ Though the aetiology
Strengths and limitations of this study

- As there has been no systematic synthesis of the evidence to inform perioperative management of people with kidney failure receiving dialysis, the scoping review method is well suited as it broadly summarises many types of literature.

- We tailored and enhanced our search strategy with the involvement of an information and database specialist.

- Our scoping search purposively used non-restrictive search terms related to our research question to ensure that the indexed and non-indexed literature was widely searched.

- Included studies will be summarised across perioperative domains of care, which will be developed post hoc and serve to inform future research avenues.

- Limitations include a search and study selection strategy that may not capture potentially relevant interventions tested in heterogeneous populations that were not limited to people receiving chronic dialysis.

of CKD is multifactorial, staging of kidney disease severity is common across types of CKD. Some people with the most severe impairment (ie, the lowest eGFR) receive kidney transplantation or dialysis as lifesustaining therapy for their kidney failure. ${ }^{1}$ People with CKD who have sustained eGFRs less than $15 \mathrm{~mL} / \mathrm{min} / 1.73 \mathrm{~m}^{2}$ and are managed with dialysis are included in the category G5D (ie, CKD G5D). ${ }^{3}$

People with CKD G5D often have concurrent chronic health conditions, many of which may be associated with the cause or consequences of their kidney disease. These co-occurring conditions can present a significant additional management burden. ${ }^{4}$ Cardiovascular (CV) disease is present in approximately $31 \%-50 \%$ of people with CKD G5D and is the leading cause of 
death in patients who receive dialysis ${ }^{5-7}$; rates of $\mathrm{CV}$ death are 10-20 times higher than in people with normal kidney function. ${ }^{8}$ Other chronic comorbidities have a similarly increased prevalence in this population. ${ }^{4}$ In addition to an increased comorbidity burden, part of their treatment burden may also include surgical procedures. Major surgery has often been defined as a procedure that requires a patient to be hospitalised for at least one night. ${ }^{9-11}$ People with CKD G5D have incident rates of major surgery up to 13 times higher than people with normal kidney function, ${ }^{12}$ and higher risks of postoperative complications, including mortality. ${ }^{13}$ For this reason, level of kidney function is included as an important predictor in many perioperative risk calculators, such as the Revised Cardiac Risk Index or the National Surgical Quality Improvement Program Surgical Risk Calculator. ${ }^{11} 14$

Perioperative medicine is the 'integrated, multidisciplinary medical care of patients from the moment of contemplation of surgery until full recovery. ${ }^{15}$ This field is rapidly evolving with research and clinical efforts directed towards addressing preoperative and intraoperative risk factors for adverse postoperative outcomes. Risk factors for postoperative mortality such as preoperative anaemia, bleeding risk, CV disease burden and fluid overload (among others) occur much more often in people with CKD G5D. ${ }^{16}$ Additionally, the risks associated with planning for dialysis therapy around the time of surgery are unique to this patient group. Despite having a higher incidence of major surgery and poorer postoperative outcomes compared with patients with normal kidney function, there is a paucity of literature that has systematically investigated the perioperative management of those with kidney failure receiving dialysis. Strategies for management in the perioperative period might involve risk stratification and preoperative risk factor optimisation, interventions to mitigate postoperative risk for those who have surgery, protocolised care for this specific population, or monitoring for complications. Scoping reviews involve highly structured searches of the literature to summarise both the breadth and depth of the available evidence for a research topic of interest. ${ }^{17}$ They are commonly used to map the literature and identify where there may be gaps in evidence for researchers to fill. ${ }^{18}$ Although there are expert-informed resources that suggest perioperative practices for clinicians to follow, we are not aware of a systematic synthesis of the perioperative evidence specific for people with kidney failure receiving dialysis.

In this manuscript we describe a protocol for a scoping review of the literature to identify the existing perioperative strategies, protocols, pathways and interventions that have been studied or implemented in people with CKD G5D undergoing major surgical procedures.

\section{METHODS AND ANALYSIS}

In conducting this scoping review, we will follow the Joanna Briggs Institute (JBI) methodology, and other expert recommended scoping review steps. ${ }^{17}{ }^{19}$ In developing this protocol we referred to the Preferred Reporting Items for Systematic Reviews and Meta-Analyses extension for scoping reviews (PRISMA-ScR), in addition to some items from the PRISMA Protocols guidelines. ${ }^{20}{ }^{21}$ Consistent with scoping review methodology, the research question for this review is intentionally broad, to enable synthesis of the breadth of available evidence (and gaps) regarding perioperative management of people with CKD G5D. The research question for this scoping review is: What perioperative strategies, protocols, pathways and interventions have been studied in or implemented for people with CKD G5D undergoing major surgical procedures?

\section{Eligibility criteria}

Eligible studies will include adults (18 years and older) with CKD G5D undergoing major surgical procedures, and must report on an approach to care for this group during the perioperative period. CKD G5D will be defined as people who receive chronic haemodialysis or peritoneal dialysis for at least 3 months, as per standard definitions. ${ }^{13}$ Major surgical procedures are typically defined as the requirement that patients stay in hospital for at least one night. ${ }^{9-11}$ However, as this definition is arbitrary, we will include all studies where authors describe the surgical interventions as major or using analogous terms or descriptors at the full-text phase of our review. Because the primary concept for this review is perioperative management of the CKD G5D population, the study must report on an approach to perioperative management and not solely on a surgical technique. Perioperative strategies in the preoperative, intraoperative and postoperative phases will be eligible for inclusion, as defined by the study. Potential studies may report on (but are not limited to) protocols, risk stratification, optimisation or detection of risk factors, monitoring strategies, interventions (ie, medications, fluids, and so on), recommendations, guidelines, best practices, care pathways or bundles. Our review is not focused on the outcome of included studies, nor is study inclusion dependent on the outcome of the study. We will include this as part of our data extraction.

Studies from all dates and languages will be eligible for inclusion. When the abstract or full text of a study is not reported in English, we will access the local scientific community who are fluent in the reported language for translation. Study type may include primary research using quantitative, qualitative, or mixed methods designs, which includes randomised interventional designs and observational studies. Additionally, conference abstracts from the last 3 years will be examined in EMBASE (see detailed section on Information Sources and Search Strategy Development). Clinical pathways or protocols, clinical practice guidelines and recommendations are also eligible, and these resources will be reviewed for the cited primary research studies that form the basis of their recommendations. We will exclude systematic, scoping, or narrative reviews, but will review identified studies of this type for their contents and citations for potential inclusion in our review. 
Studies in children, or non-human subjects are ineligible. Studies that investigate perioperative strategies for people on dialysis as a result of an acute kidney injury will not be eligible. Diagnostic procedures, those that are usually performed as an outpatient, or by interventional radiology (insertion of dialysis central venous catheters) will be excluded. Studies in patients receiving an incident kidney transplant (ie, from dialysis to transplant) will be eligible for inclusion, but studies focused on major surgery for people who were previously transplanted and no longer on dialysis will be excluded. Peritransplant immunosuppression is not the focus of this review, and so these studies will be excluded as well.

\section{Information sources and search strategy development}

The search strategy for this review was developed using Medical Subject Headings and text words from titles and abstracts based on three key domains: (1) perioperative period, (2) strategies, protocols, interventions, and so on, and (3) CKD receiving dialysis. The initial draft search was reviewed by a health sciences librarian with expertise in scoping reviews (Dr Diane Lorenzetti) and is included in online supplemental appendix 1. In order to develop a rigorous search strategy, clinician and methodological experts from our research group (TGH, BRH, PER) will first test our key search terms in MEDLINE (Ovid interface, 1946 to present) to ensure adequate sensitivity and specificity of our strategy. Once the strategy is tested in MEDLINE, similar searches will be performed in EMBASE, CINAHL Plus, Cochrane Database of Systematic Reviews, and Cochrane Controlled Trials Registry for published literature from inception date of these databases, respectively. Conference abstracts for the past 3 years will also be included in our search of the EMBASE database. Google Scholar will be used to review citations of included articles after full-text inclusion for additional literature, and other reviews (narrative, systematic, and so on) will be included at the abstract phase and references reviewed in the full-text phase for inclusion. We will search PROSPERO for registered systematic reviews of similar topics (PROSPERO does not accept scoping review protocol registration at this time). After conducting the initial peer-reviewed literature search, we will develop a grey literature strategy as per the Canadian Agency for Drugs and Technologies in Health $(\mathrm{CADTH})$ guidelines to look for published care bundles and pathways. ${ }^{22} \mathrm{We}$ will use the CADTH recommendations to search online search engines such as Google Canada/USA/UK, and will review the first 100 website links on each search engine. Additionally, we will review clinical trials databases for registered protocols and dissertation theses repositories (through the Proquest database on the University of Calgary library website), and medical reference websites for relevant recommendations. We anticipate completing our search in February 2020.

\section{Study selection process}

Three study reviewers with training on the study protocol and eligibility criteria will participate in study selection for this scoping review (TGH, JFF, CO). The reviewers have methodological expertise in systematic literature searches, and TGH as the primary reviewer and a nephrologist has content expertise in this topic. Covidence software will be used to remove duplicate articles once all database searches are combined, and complete abstract and full-text searches (https://www.covidence. $\mathrm{org} /$ ). Covidence is an internet-based software that allows for distribution of studies among reviewers for systematic and scoping reviews and streamlines the inclusion/exclusion of studies at each stage of the review. Each identified study or resource will be reviewed independently and in duplicate throughout all phases of this review. All studies will have their title and abstract reviewed for inclusion, which will be focused on including studies that are likely to have included patients with CKD G5D (although does not have to explicitly mention dialysis), around the time of surgery. At this stage, we will include studies for which the abstract is unclear whether or not they focused on perioperative strategies instead of a surgical approach. We will screen the first 100 abstracts in triplicate as a pilot for reviewer calibration. If any study is identified by at least one reviewer for inclusion, it will be propagated to the next phase of our review even if there is no consensus between both reviewers.

The full-text phase of the scoping review will be independently completed in duplicate. If there is disagreement about study inclusion at this stage, a third reviewer's opinion will resolve the lack of consensus. Reference lists of included studies will be reviewed to identify additional studies. Additionally, references of websites or online resources will be reviewed for inclusion. The fulltext review will apply more stringent criteria to exclude studies focused on the primary surgical approach and non-perioperative strategies, or if it was not clear that people with CKD G5D were included. Grey literature sources will be reviewed in similar detail as the rest of the full-text review.

\section{Data extraction process}

We have generated a data extraction spreadsheet as adapted from general recommendations from the JBI and the Template for Intervention Description and Replication (TIDieR) checklist. ${ }^{19} 23$ Table 1 includes a list of the variables we will be extracting from included studies. This includes data related to study type, patient characteristics, intervention/procedure and outcomes. Study data of interest will include year of publication, journal or website, country of origin, study design, number of participants and length of follow-up. Number of patients who were lost to follow-up will be abstracted as well. Patient characteristics of interest will include age, sex, proportion with comorbidities of perioperative importance, body mass index, baseline eGFR (if not all the study population were receiving dialysis), proportion of patients with CKD G5D (and type of dialysis if included) and proportion of patients who are kidney transplant candidates. Intervention type/dosage will be abstracted, including timing 


\begin{tabular}{|c|c|}
\hline Category & Variable \\
\hline $\begin{array}{l}\text { Study } \\
\text { demographics }\end{array}$ & $\begin{array}{l}\text { Author last name (first author) } \\
\text { Year } \\
\text { Title } \\
\text { Journal or source } \\
\text { Country of origin } \\
\text { Study design }\end{array}$ \\
\hline Study population & $\begin{array}{l}\text { Start number (n) } \\
\text { Completed (n) } \\
\text { Completed (\%) } \\
\text { Age (mean; years) } \\
\text { Age (SD; years) } \\
\text { Age (median; years) } \\
\text { Age (IQR; years) } \\
\text { Age (Q1; years) } \\
\text { Age (Q3; years) } \\
\text { Male (n) } \\
\text { Male (\%) } \\
\text { DM (\%) } \\
\text { CVD (\%) } \\
\text { CVD definition } \\
\text { HTN (\%) } \\
\text { Smoking (\%) } \\
\text { Malignancy history (\%) } \\
\text { Lung disease (\%) } \\
\text { Chronic liver disease (\%) } \\
\text { BMI (mean) } \\
\text { BMI (SD) } \\
\text { Baseline eGFR (mL/min/1.73 } \text { m }^{2} \text { ) - if some of the } \\
\text { study population are not on dialysis } \\
\text { Dialysis (n) } \\
\text { Dialysis (\%) } \\
\text { Dialysis vintage (years) } \\
\text { HD (n) } \\
\text { HD (\%) } \\
\text { PD (n) } \\
\text { PD (\%) } \\
\text { Kidney transplant eligible/candidate ( } n \text { ) } \\
\text { Kidney transplant eligible/candidate (\%) }\end{array}$ \\
\hline $\begin{array}{l}\text { Procedure details } \\
\text { (as specified) }\end{array}$ & $\begin{array}{l}\text { Types of surgeries (and number/\% of each) } \\
\text { Emergent }(n) \\
\text { Emergent }(\%) \\
\text { Urgent }(n) \\
\text { Urgent }(\%) \\
\text { Semiurgent }(n) \\
\text { Semiurgent }(\%) \\
\text { Elective }(n) \\
\text { Elective }(\%) \\
\text { Length of procedure } \\
\text { Anaesthetic type }\end{array}$ \\
\hline $\begin{array}{l}\text { Intervention } \\
\text { details }\end{array}$ & $\begin{array}{l}\text { Intervention description (why, what, who } \\
\text { provided, how, where, when and how much, } \\
\text { tailoring and modifications, how well-per } \\
\text { TIDieR) } \\
\text { Timing of intervention before surgery (specific) } \\
\text { Timing of intervention (categorical: preoperative, } \\
\text { intraoperative, postoperative) } \\
\text { Outcome being assessed (if applicable) } \\
\text { Length of outcome surveillance (days) } \\
\text { Patients with outcome in intervention arm (n) } \\
\text { Patients with outcome in intervention arm (\%) } \\
\text { Measure of efficacy or effectiveness (if } \\
\text { applicable, and as positive/negative as reported) }\end{array}$ \\
\hline Notes & Extra notes for the study of importance \\
\hline
\end{tabular}

Continued

\section{Table 1 Continued}

\section{Category Variable}

This table represents the planned data elements that we will extract in our review. This may change after review of the literature, but for now this represents the important variables determined by our study team a priori.

BMI, body mass index; CVD, cardiovascular disease; DM, Diabetes Mellitus; eGFR, estimated glomerular filtration rate; HD, haemodialysis; HTN, hypertension; PD, peritoneal dialysis; Q1, quartile 1; Q3, quartile 3; TIDieR, Template for Intervention Description and Replication.

of intervention prior to procedure (if applicable) and duration of treatment. Whether trials used a placebo or sham intervention will be included. Surgical procedure type if specified (or proportion of surgical type if more than one was included), anaesthetic type and length of procedure will be determined. Though definitions of surgical urgency are often specific to institutions and heterogeneous, ${ }^{24}$ we will extract study-defined procedure urgency such as emergent, urgent, semiurgent and elective, if provided. Examples of these types of procedures are found in the Canadian Cardiovascular Society perioperative guidelines. ${ }^{9}$ The proportion of patients who had study-specified outcomes will be extracted, in addition to length of surveillance for outcomes. For studies that evaluated an intervention or discuss an intervention, we will use TIDieR elements to extract detailed intervention information. We will extract the reported efficacy or effectiveness of each intervention if available, for inclusion as descriptive information about the intervention. If recommendations (such as on UpToDate.com or other electronic medical references) are provided without citation, this will be abstracted as well. If the included study is a conference abstract or is missing key variables, we will attempt to contact the authors to obtain additional information.

We will test our data extraction form by completing the extraction of five randomly chosen studies prior to reviewing the results as a study team, to determine whether the extraction form was comprehensive enough to meet review objectives and all included study types. Data extraction will be completed in duplicate, with one reviewer cross-checking the initial reviewer extraction from included studies.

\section{Reporting and synthesis of results}

Reporting of this scoping review will be per the PRISMA-ScR and the JBI recommendations. ${ }^{19} 20$ The synthesis will be descriptive, reporting on the number of studies included at each stage, as well as reasons for exclusion at the full-text review stage in a PRISMA flow diagram (figure 1). We will tabulate study characteristics to outline the distribution of included studies by year of publication, country of origin and surgical procedure type. Further, we will narratively report on and include a summary table outlining the strategies employed, stratified into major perioperative management domains with the following as possible categories: bleeding reduction 


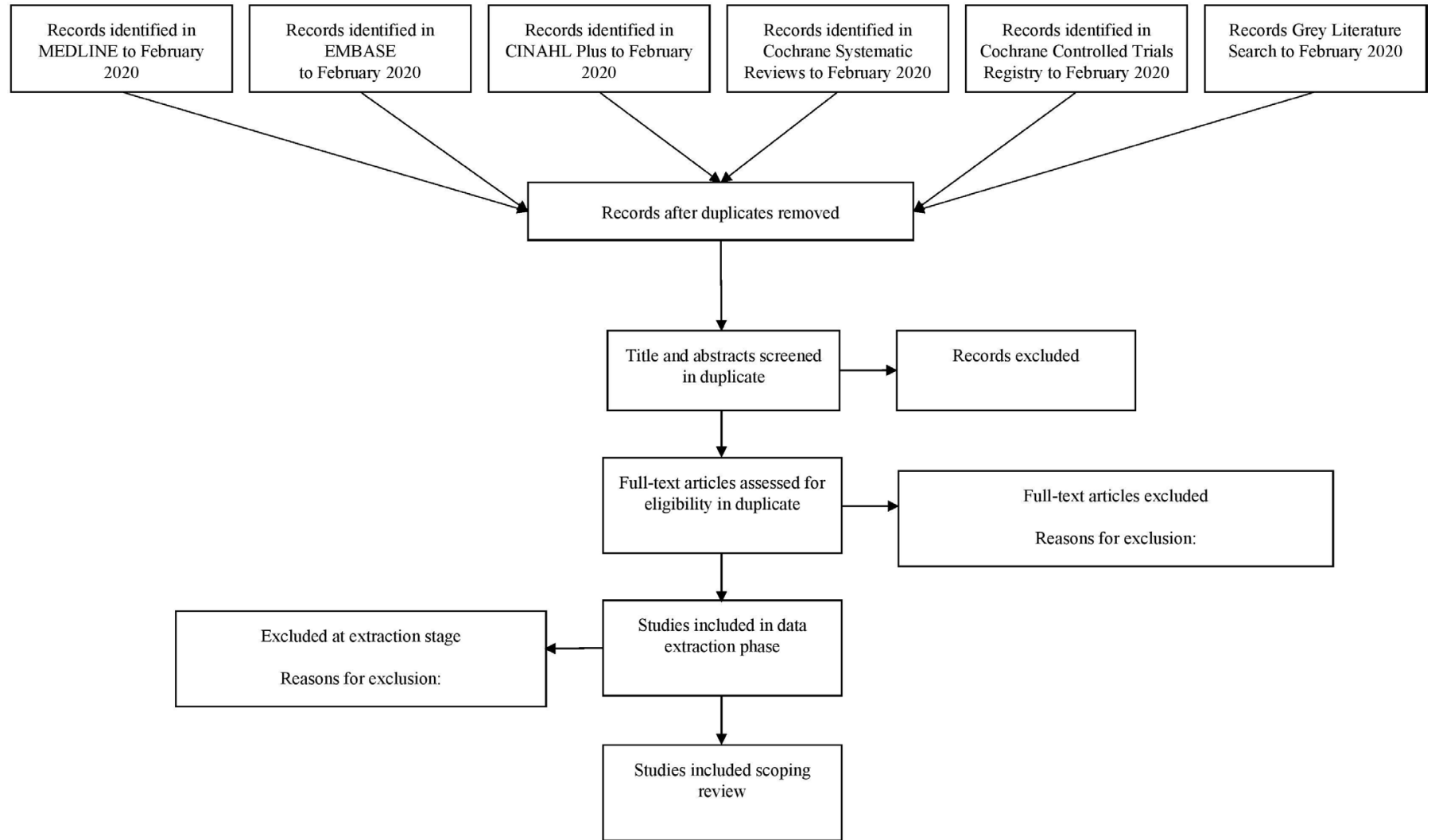

Figure 1 Flow diagram for inclusion and exclusion of studies at various stages of review. This figure represents the flow diagram for our scoping review, from the data sources through to final inclusion. This is in adherence with recommendations from the Preferred Reporting Items for Systematic Reviews and Meta-Analyses extension for scoping reviews (PRISMA-ScR). ${ }^{20}$

(ie, pharmacological and non-pharmacological), CV risk reduction, thromboembolic considerations, infection reduction, hypotension avoidance strategies and dialysis strategies. These and other possible categories will be developed post hoc once studies are identified. Within each domain, we will summarise the included studies in terms of the number of studies and people included, breadth of research and, if possible, identify gaps in the literature based on these domains. It is possible that one study may be included in more than one perioperative domain, and if so, we will modify the graphical presentation of our main summary table to allow for this. Risk of bias and quality assessment are not typically included in scoping review methods.

\section{ETHICS AND DISSEMINATION}

As this review includes previously published information or those available in the public domain, there are no applicable ethical considerations. After this review is completed, findings will be disseminated at perioperative and kidney disease-related conferences and submitted to peer-reviewed journals to reach the appropriate target audience.

\section{LIMITATIONS}

Our search and study selection strategy may limit inclusion of studies of interventions tested in heterogeneous populations that were not limited to people with kidney disease. It is possible that the potentially missed interventions were tested on some people with CKD G5D. To ameliorate this risk, we are purposively including studies from the abstract review phase that include the broader population of people with CKD and will review their full text to assess CKD G5D population inclusion. There are also limitations inherent to scoping review methodology as well, as there is no formal risk of bias assessment or quality assessment included. However, these assessments are not the purpose of scoping reviews as we are trying to map available literature and current gaps in evidence (and not quantitatively assess the evidence at this stage). Despite these limitations, we will be completing an extensive search of the published peer-reviewed literature and the grey literature sources which enhances the rigour of our planned review. Gaps identified in this review will inform future research avenues in the perioperative management of patients with kidney failure receiving dialysis.

\section{Author affiliations}

${ }^{1}$ Department of Medicine, University of Calgary Cumming School of Medicine, Calgary, Alberta, Canada

${ }^{2}$ Department of Community Health Sciences, University of Calgary Cumming School of Medicine, Calgary, Alberta, Canada 
${ }^{3}$ Division of Nephrology, Department of Medicine, University of Alberta Faculty of Medicine and Dentistry, Edmonton, Alberta, Canada

${ }^{4}$ O'Brien Institute for Public Health, University of Calgary Cumming School of Medicine, Calgary, Alberta, Canada

${ }^{5}$ Libin Cardiovascular Institute, University of Calgary Cumming School of Medicine, Calgary, Alberta, Canada

Twitter Tyrone G Harrison @tharrisonmd, Janine F Farragher @janinef and Shannon M Ruzycki @ShannonRuzyck

Acknowledgements We would like to thank Dr Diane Lorenzetti for her expert assistance with reviewing our search strategy.

Contributors TGH and BRH conceived the study. TGH outlined the protocol, drafted this manuscript and is the guarantor of the protocol manuscript. TGH, BRH, JFF, CO, MD, MTJ, DM, SMR, KBZ and PER jointly developed the research questions and revised the paper. All authors approved the final version for submission.

Funding TGH is supported by a Kidney Research Scientist Core Education and National Training Program (KRESCENT) postdoctoral fellowship (cosponsored by the Kidney Foundation of Canada and the Canadian Institutes of Health Research (CIHR)) and the Clinician Investigator Program at the University of Calgary. JFF is supported by a KRESCENT postdoctoral fellowship and a CIHR Fellowship. MTJ is supported by a ClHR New Investigator Award and is the principal investigator of an investigator-initiated research grant from Amgen, Canada, which is not related to this work. BRH is supported by the Roy and Vi Baay Chair in Kidney Research. PER is supported by a CIHR Project Grant.

Competing interests None declared.

Patient and public involvement Patients and/or the public were not involved in the design, conduct, reporting, or dissemination plans of this research.

Patient consent for publication Not required.

Provenance and peer review Not commissioned; externally peer reviewed.

Open access This is an open access article distributed in accordance with the Creative Commons Attribution Non Commercial (CC BY-NC 4.0) license, which permits others to distribute, remix, adapt, build upon this work non-commercially, and license their derivative works on different terms, provided the original work is properly cited, appropriate credit is given, any changes made indicated, and the use is non-commercial. See: http://creativecommons.org/licenses/by-nc/4.0/.

ORCID IDs

Tyrone G Harrison http://orcid.org/0000-0003-1068-8673

Shannon M Ruzycki http://orcid.org/0000-0002-8122-2910

\section{REFERENCES}

1 Kidney disease: improving global outcomes CKD Work Group. KDIGO 2012 clinical practice guideline for the evaluation and management of chronic kidney disease. Kidney Int Supp 2013;3.

2 Saran R, Robinson B, Abbott KC, et al. Us renal data system 2017 annual data report: epidemiology of kidney disease in the United States. Am J Kidney Dis 2018;71:A7.

3 Levey AS, Eckardt K-U, Dorman NM, et al. Nomenclature for kidney function and disease: report of a kidney disease: improving global outcomes (KDIGO) consensus conference. Kidney Int 2020;97:1117-29.

4 Fraser SDS, Taal MW. Multimorbidity in people with chronic kidney disease: implications for outcomes and treatment. Curr Opin Nephrol Hypertens 2016;25:465-72.
5 Goodkin DA, Bragg-Gresham JL, Koenig KG, et al. Association of comorbid conditions and mortality in hemodialysis patients in Europe, Japan, and the United States: the dialysis outcomes and practice patterns study (DOPPS). J Am Soc Nephrol 2003;14:3270-7.

6 Gomez AT, Kiberd BA, Royston JP, et al. Comorbidity burden at dialysis initiation and mortality: a cohort study. Can J Kidney Health Dis 2015;2:68.

7 United States Renal Data System. USRDS annual data report: epidemiology of kidney disease in the United States, 2019. Available: https://www.usrds.org/annual-data-report/

8 Foley RN, Parfrey PS, Sarnak MJ. Epidemiology of cardiovascular disease in chronic renal disease. J Am Soc Nephrol 1998;9:S112-9.

9 Duceppe E, Parlow J, MacDonald P, et al. Canadian cardiovascular Society guidelines on perioperative cardiac risk assessment and management for patients who undergo noncardiac surgery. Can $J$ Cardiol 2017;33:17-32.

10 Botto F, Alonso-Coello P, Chan MTV, et al. Myocardial injury after noncardiac surgery: a large, international, prospective cohort study establishing diagnostic criteria, characteristics, predictors, and $30-$ day outcomes. Anesthesiology 2014;120:564-78.

11 Lee TH, Marcantonio ER, Mangione CM, et al. Derivation and prospective validation of a simple index for prediction of cardiac risk of major noncardiac surgery. Circulation 1999;100:1043-9.

12 Harrison T, James MT, Zarnke KB, et al. Estimated glomerular filtration rate and incidence of major surgery: a population-based cohort study. Washington, DC, USA: American Society of Nephrology Kidney Week, 2019.

13 Mooney JF, Ranasinghe I, Chow CK, et al. Preoperative estimates of glomerular filtration rate as predictors of outcome after surgery: a systematic review and meta-analysis. Anesthesiology 2013;118:809-24.

14 American College of Surgeons. Acs NSQIP surgical risk calculator, 2019. Available: http://www.riskcalculator.facs.org/RiskCalculator/ index.jsp

15 Grocott MPW, Edwards M, Mythen MG, et al. Peri-operative care pathways: re-engineering care to achieve the 'triple aim'. Anaesthesia 2019;74 Suppl 1:90-9.

16 Nasr R, Chilimuri S. Preoperative evaluation in patients with endstage renal disease and chronic kidney disease. Health Serv Insights 2017; 10:117863291771302.

17 Levac D, Colquhoun H, O'Brien KK. Scoping studies: advancing the methodology. Implement Sci 2010;5:69.

18 Nyanchoka L, Tudur-Smith C, Thu VN, et al. A scoping review describes methods used to identify, prioritize and display gaps in health research. J Clin Epidemiol 2019:109:99-110.

19 Peters MDJ, Godfrey CM, Khalil H, et al. Guidance for conducting systematic scoping reviews. Int J Evid Based Healthc 2015;13:141-6.

20 Tricco AC, Lillie E, Zarin W, et al. PRISMA extension for scoping reviews (PRISMA-ScR): checklist and explanation. Ann Intern Med 2018;169:467-73.

21 Shamseer L, Moher D, Clarke M, et al. Preferred reporting items for systematic review and meta-analysis protocols (PRISMA-P) 2015: elaboration and explanation. BMJ 2015;350:g7647.

22 Canadian Agency for Drugs and Technologies in Health (CADTH). Grey matters: a practical search tool for evidence-based medicine, 2019. Available: https://www.cadth.ca/resources/finding-evidence/ grey-matters.

23 Hoffmann TC, Glasziou PP, Boutron I, et al. Better reporting of interventions: template for intervention description and replication (TIDieR) checklist and guide. BMJ 2014;348:g1687.

24 Schwarze ML, Barnato AE, Rathouz PJ, et al. Development of a list of high-risk operations for patients 65 years and older. JAMA Surg 2015;150:325-31. 